Chiral chromatographic isolation on milligram scale of the human African trypanosomiasis treatment D- and L-eflornithine

Mikael Boberg ${ }^{1}$, Anna C. Jonson ${ }^{2}$, Hanna Leek ${ }^{2}$, Rasmus Jansson-Löfmark ${ }^{1,3}$ and Michael Ashton $^{1 *}$

${ }^{1}$ Unit for Pharmacokinetics and Drug Metabolism, Sahlgrenska Academy, University of Gothenburg, Gothenburg, Sweden

${ }^{2}$ Early Chemical Development, Pharmaceutical Sciences, BioPharmaceuticals R\&D, AstraZeneca, Gothenburg, Sweden

${ }^{3}$ DMPK, Research and Early Development Cardiovascular, Renal and Metabolism, BioPharmaceuticals R\&D, AstraZeneca, Gothenburg, Sweden

${ }^{*}$ Corresponding author: Professor Michael Ashton, Unit for Pharmacokinetics and Drug Metabolism, Sahlgrenska Academy, University of Gothenburg, Box 431 SE-405 30, Gothenburg, Sweden. Email: michael.ashton@gu.se 
(D)

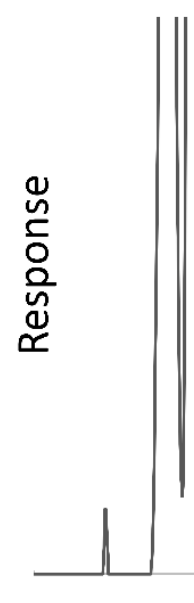

0

\section{Time (min)}

Supplementary Figure S1. Milligram scale HPLC isolation of D- and L-eflornithine using Chirobiotic TAG $(20 \times 250 \mathrm{~mm}, 5 \mu \mathrm{m})$ with buffer:EtOH 75:25 (v/v\%) (buffer containing $17 \mathrm{mM} \mathrm{AcOH}$ and $2 \mathrm{mM}$ TEA) at ambient temperature, $18 \mathrm{~mL} / \mathrm{min}$ with $10 \mathrm{mg}$ of racemic sample $(30 \mathrm{mg} / \mathrm{mL}$ (aq.)) injected and detected with ELSD. 
Supplementary Table S1. Chiral stationary phase and modifier combinations evaluated for Dand $L$-eflornithine and the eflornithine-OPA derivate in SFC mode.

\begin{tabular}{|c|c|c|c|c|c|c|c|}
\hline \multicolumn{3}{|c|}{ For D- and L-eflornithine } & & \multicolumn{4}{|c|}{ For D- and L-eflornithine-OPA derivate } \\
\hline Chiralpak IA & K'Peak 1 & $\alpha$ & $\mathrm{R}_{\mathrm{s}}$ & Chiralpak IA & K'Peak 1 & $\alpha$ & $\mathrm{R}_{\mathrm{s}}$ \\
\hline a & 1.3 & 1 & $\mathrm{~N} / \mathrm{A}$ & a & 5.8 & 1 & $\mathrm{~N} / \mathrm{A}$ \\
\hline b & 0.8 & 1 & $\mathrm{~N} / \mathrm{A}$ & b & 4.2 & 1 & $\mathrm{~N} / \mathrm{A}$ \\
\hline Chiralpak IB & & & & c & No peak & N/A & N/A \\
\hline a & 1.2 & 1 & $\mathrm{~N} / \mathrm{A}$ & Chiralpak IB & & & \\
\hline $\begin{array}{l}\text { b } \\
\text { Chiralpak IC }\end{array}$ & 0.7 & 1 & $\mathrm{~N} / \mathrm{A}$ & $\begin{array}{l}a \\
b\end{array}$ & $\begin{array}{l}\text { No Peak } \\
4.5\end{array}$ & $\begin{array}{l}N / A \\
1\end{array}$ & $\begin{array}{l}\text { N/A } \\
\text { N/A }\end{array}$ \\
\hline a & 0.9 & 1 & $\mathrm{~N} / \mathrm{A}$ & c & No peak & $\mathrm{N} / \mathrm{A}$ & $\mathrm{N} / \mathrm{A}$ \\
\hline$b$ & 0.7 & 1 & $\mathrm{~N} / \mathrm{A}$ & Chiralpak IC & & & \\
\hline Chiralpak ID & & & & a & $\mathrm{RT}: 4.7$ & 1 & $\mathrm{~N} / \mathrm{A}$ \\
\hline $\mathrm{a}$ & 0.7 & 1 & $\mathrm{~N} / \mathrm{A}$ & $\mathrm{b}$ & No peak & N/A & $\mathrm{N} / \mathrm{A}$ \\
\hline $\mathrm{b}$ & 0.7 & 1 & $\mathrm{~N} / \mathrm{A}$ & c & No peak & N/A & $\mathrm{N} / \mathrm{A}$ \\
\hline Chiralpak IE & & & & Chiralpak ID & & & \\
\hline a & 2.2 & 1 & $\mathrm{~N} / \mathrm{A}$ & a & 5.3 & 1.04 & ND \\
\hline b & 0.9 & 1 & $\mathrm{~N} / \mathrm{A}$ & b & 4.4 & 1.03 & ND \\
\hline $\begin{array}{l}\text { d } \\
\text { Chiralpak AD }\end{array}$ & No peak & $\mathrm{N} / \mathrm{A}$ & $\mathrm{N} / \mathrm{A}$ & $\begin{array}{l}\text { C } \\
\text { Chiralpak AS }\end{array}$ & No peak & N/A & $\mathrm{N} / \mathrm{A}$ \\
\hline a & 0.6 & 1 & $\mathrm{~N} / \mathrm{A}$ & $\mathrm{a}$ & 3.9 & 1.09 & ND \\
\hline$b$ & 0.6 & 1 & $\mathrm{~N} / \mathrm{A}$ & b & 2.6 & 1.09 & ND \\
\hline Chiralpak AS & & & & $\mathrm{c}$ & 5.5 & 1 & N/A \\
\hline a & 0.7 & 1 & $\mathrm{~N} / \mathrm{A}$ & (S,S)Whelk-01 & & & \\
\hline b & 1.6 & 1 & $\mathrm{~N} / \mathrm{A}$ & $\mathrm{a}$ & 5.2 & 1 & N/A \\
\hline c & 1.6 & 1 & $\mathrm{~N} / \mathrm{A}$ & b & 4.1 & 1 & N/A \\
\hline Lux C3 & & & & c & No peak & $\mathrm{N} / \mathrm{A}$ & $\mathrm{N} / \mathrm{A}$ \\
\hline a & 2.4 & 1 & $\mathrm{~N} / \mathrm{A}$ & Chiralpak IG & & & \\
\hline b & 0.9 & 1 & $\mathrm{~N} / \mathrm{A}$ & aDEA & 4.4 & 1.2 & 1.5 \\
\hline Lux C4 & & & & a $5-40 \%$ & 3.7 & 1.03 & ND \\
\hline a & 4.2 & 1 & $\mathrm{~N} / \mathrm{A}$ & b & 2.9 & 1 & $\mathrm{~N} / \mathrm{A}$ \\
\hline $\begin{array}{l}\text { b } \\
\text { (S,S)Whelk-01 }\end{array}$ & 1.5 & 1 & $\mathrm{~N} / \mathrm{A}$ & $\begin{array}{l}\text { C } \\
\text { Kromasil CelluCoat }\end{array}$ & No peak & $\mathrm{N} / \mathrm{A}$ & $\mathrm{N} / \mathrm{A}$ \\
\hline a & 1.5 & 1 & $\mathrm{~N} / \mathrm{A}$ & $a$ & No peak & $\mathrm{N} / \mathrm{A}$ & $\mathrm{N} / \mathrm{A}$ \\
\hline $\mathrm{b}$ & 0.9 & 1 & $\mathrm{~N} / \mathrm{A}$ & b & No peak & N/A & $\mathrm{N} / \mathrm{A}$ \\
\hline tBuCQN & & & & c & No peak & N/A & $\mathrm{N} / \mathrm{A}$ \\
\hline $\mathrm{a}$ & 8.7 & 1 & $\mathrm{~N} / \mathrm{A}$ & Lux A1 & & & \\
\hline$b$ & 2.2 & 1 & $\mathrm{~N} / \mathrm{A}$ & a & 3.9 & 1.05 & ND \\
\hline $\mathrm{c}$ & No peak & $\mathrm{N} / \mathrm{A}$ & $\mathrm{N} / \mathrm{A}$ & b & 3.1 & 1.07 & ND \\
\hline Chirobiotic T & & & & $\mathrm{c}$ & 5.0 & 1 & N/A \\
\hline a & No peak & $\mathrm{N} / \mathrm{A}$ & $\mathrm{N} / \mathrm{A}$ & Chiralpak IB N-3 & & & \\
\hline $\mathrm{b}$ & 3.6 & 1 & $\mathrm{~N} / \mathrm{A}$ & a & 4.3 & 1 & $\mathrm{~N} / \mathrm{A}$ \\
\hline d & 6.9 & 1 & $\mathrm{~N} / \mathrm{A}$ & b & 3.2 & 1 & $\mathrm{~N} / \mathrm{A}$ \\
\hline Chirobiotic V2 & & & & $\mathrm{c}$ & 5.7 & 1 & $\mathrm{~N} / \mathrm{A}$ \\
\hline a & No peak & $\mathrm{N} / \mathrm{A}$ & $\mathrm{N} / \mathrm{A}$ & Chiralcel OJ & & & \\
\hline b & 8.6 & 1 & $\mathrm{~N} / \mathrm{A}$ & b & No peak & N/A & $\mathrm{N} / \mathrm{A}$ \\
\hline Chiralpak IG & & & & $\mathrm{c}$ & No peak & $\mathrm{N} / \mathrm{A}$ & $\mathrm{N} / \mathrm{A}$ \\
\hline a & No peak & $\mathrm{N} / \mathrm{A}$ & $\mathrm{N} / \mathrm{A}$ & Chiralart Cellulose SJ & & & \\
\hline $\mathrm{b}$ & No peak & $\mathrm{N} / \mathrm{A}$ & $\mathrm{N} / \mathrm{A}$ & a & 3.3 & 1 & $\mathrm{~N} / \mathrm{A}$ \\
\hline & & & & $\mathrm{b}$ & 2.3 & 1 & $\mathrm{~N} / \mathrm{A}$ \\
\hline & & & & $\mathrm{c}$ & 4.7 & 1 & $\mathrm{~N} / \mathrm{A}$ \\
\hline
\end{tabular}


Supplementary Table S2. Chiral stationary phase and modifier combinations evaluated for D- and L-eflornithine in HPLC mode.

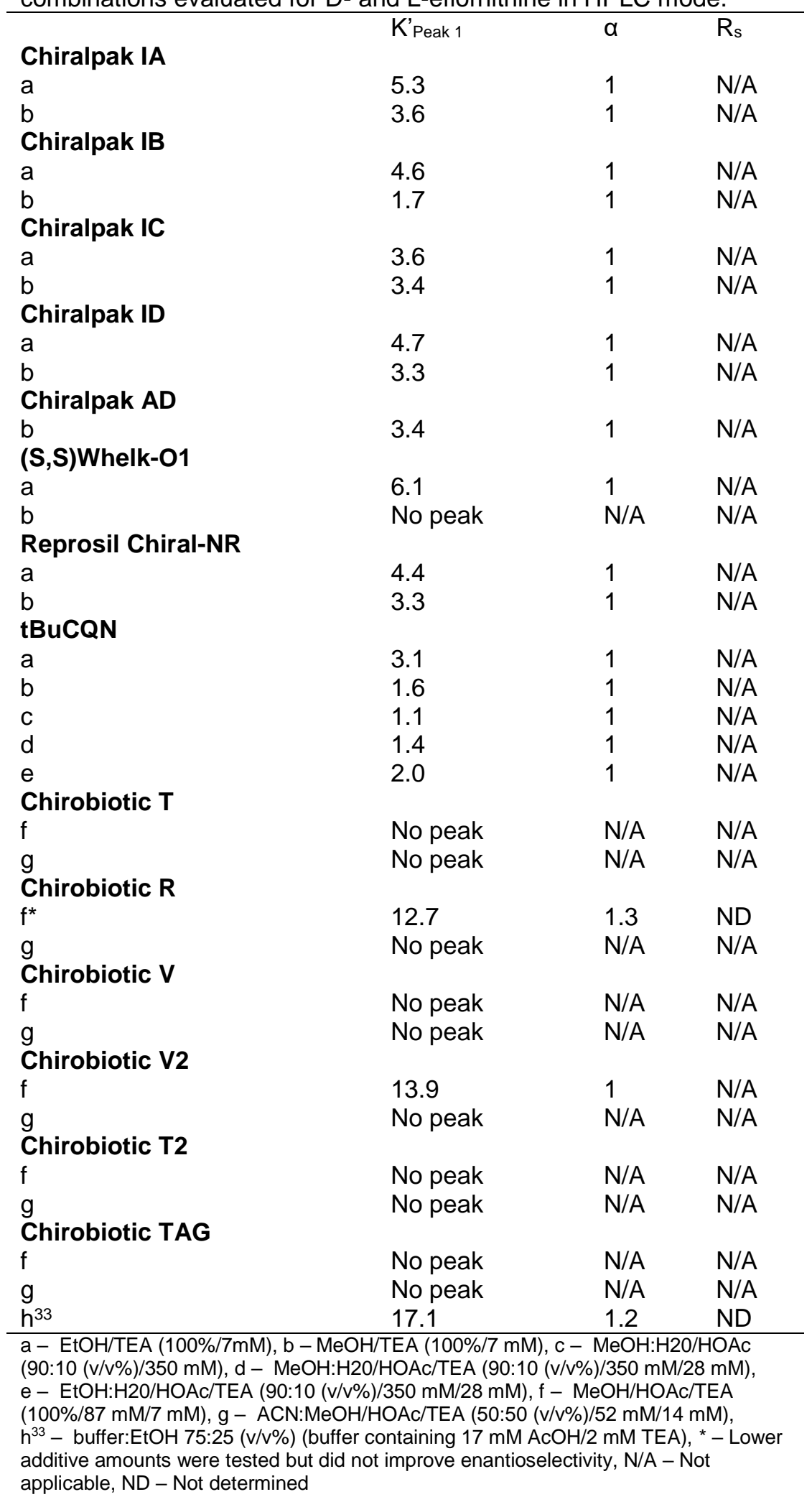

